\title{
Controlled Self-Organization of Atom Vacancies in Monatomic Gallium Layers
}

\author{
P. C. Snijders, ${ }^{1, *}$ E. J. Moon, ${ }^{2}$ C. González,${ }^{3}$ S. Rogge, ${ }^{1}$ J. Ortega,${ }^{3}$ F. Flores, ${ }^{3}$ and H. H. Weitering ${ }^{2,4}$ \\ ${ }^{1}$ Kavli Institute of NanoScience Delft, Delft University of Technology, 2628 CJ Delft, The Netherlands* \\ ${ }^{2}$ Department of Physics and Astronomy, The University of Tennessee, Knoxville, Tennessee 37996, USA \\ ${ }^{3}$ Departamento de Física Teórica de la Materia Condensada, Universidad Autónoma, 28049 Madrid, Spain \\ ${ }^{4}$ Materials Science and Technology Division, Oak Ridge National Laboratory, Oak Ridge, Tennessee 37831, USA
}

(Received 10 May 2007; published 14 September 2007)

\begin{abstract}
$\mathrm{Ga}$ adsorption on the $\mathrm{Si}(112)$ surface results in the formation of pseudomorphic $\mathrm{Ga}$ atom chains. Compressive strain in these atom chains is relieved via creation of adatom vacancies and their selforganization into meandering vacancy lines. The average spacing between these line defects can be controlled, within limits, by adjusting the chemical potential $\mu$ of the Ga adatoms. We derive a lattice model that quantitatively connects density functional theory (DFT) calculations for perfectly ordered structures with the fluctuating disorder seen in experiment and the experimental control parameter $\mu$. This hybrid approach of lattice modeling and DFT can be applied to other examples of line defects in heteroepitaxy.
\end{abstract}

DOI: 10.1103/PhysRevLett.99.116102

Many physical, chemical, and biological phenomena are manifestations of self-organization of matter, such as crystal growth, protein folding, or formation of galaxy clusters. Well-known examples of self-organization in advanced materials systems include the formation of magic metal clusters, pyramid quantum dots, quantum dot superlattices in heterostructures [1], and the ordering of atom vacancies into line defects or vacancy line superstructures [2-4]. For application purposes, it would be essential to control the size, uniformity, and spacing of such nano-objects. This goes against the odds of thermodynamic fluctuations that are especially profound in low-dimensional systems, and the stochastic nature of nucleation and growth.

Here, we show that the average spacing between VLs in a monatomic Ga layer on $\mathrm{Si}(112)$ can be controlled and varied continuously, within limits, via the chemical potential of the adsorbate species, $\mu$. Ga vacancies self-organize into a $n \times 1 \mathrm{VL}$ superstructure [Fig. 1(a)-1(c)], similar to the formation of the well-known $n \times 2$ superstructures for $\mathrm{Ge}$ on $\mathrm{Si}(100)$ [2-4]. Entropic fluctuations compete with this ordering process, however, resulting in meandering VLs where the average line spacing is fixed by the chemical potential. The meandering amplitude is limited by elastic repulsions between VLs. Such a two-dimensional (2D) interacting vacancy line system has been modeled as a 1D random walker trapped in a harmonic potential representing the collective mean field of all the other VLs [4]. For systems such as $\mathrm{Ge}$ on $\mathrm{Si}(100)$, this 1D model seems to capture the observed meandering quite well, allowing for a straightforward determination of the kink energy and line repulsion from statistical analysis of fluctuations in Scanning Tunneling Microscopy (STM) images. Step fluctuations on vicinal crystal surfaces have been analyzed in similar fashion, using continuum modeling [5].

An interesting question is how the constraints imposed by the discreteness of a lattice modifies the elastic interactions that are driving the self-organization of vacancies,
PACS numbers: 68.35.-p, 05.65.+b, 68.37.Ef, 81.16.Dn

particularly for short VL spacings. Discrete VL spacings conceivably lead to significant vacancy-vacancy correlations that cannot be captured by the usual mean field or continuum models. Ga on $\mathrm{Si}(112)$ turns out to be an interesting test case. By tuning the average line spacing between $n=5$ and $n=6$ (see Fig. 1) we find that the discrete distribution of VL spacings does not follow the scaling behavior predicted by the mean field model, and that correlations cannot be ignored. Guided by these experimental observations, we construct a lattice model in which the surface is represented as an entropic mixture or solid solution of different $m \times 1$ building blocks (henceforth $m$ will be reserved for integers and $n$ for the average periodicity). The model naturally takes into account the discrete nature of the VL spacing and contains only two parameters: (1) the chemical potential of the adsorbate

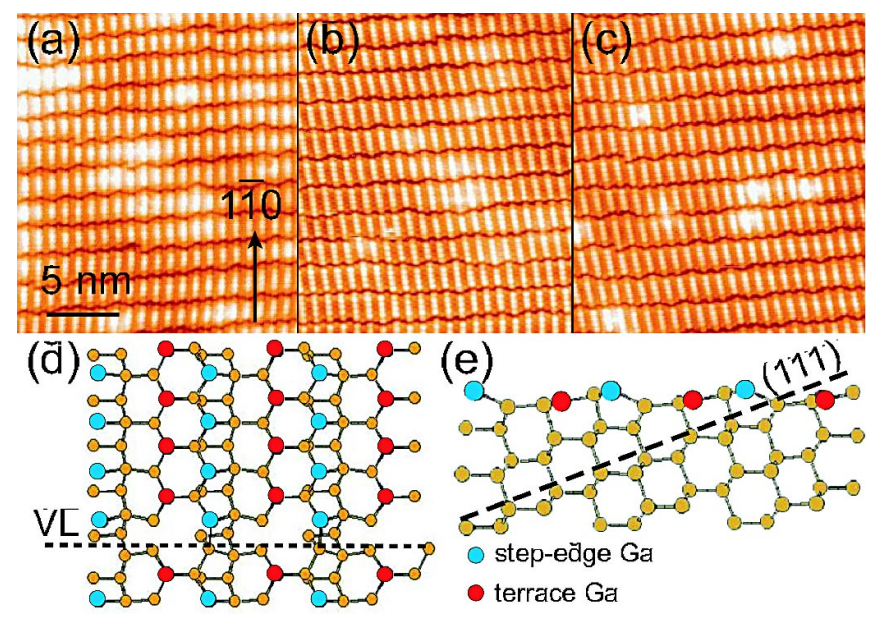

FIG. 1 (color online). (a)-(c) STM images of the Ga-covered $\operatorname{Si}(112) n \times 1 \quad$ surface: (a) $n=5.49$, (b) $n=5.68$, and (c) $n=5.88$. (d) Topview and (e) sideview of a ball and stick representation of the $\mathrm{Si}(112) 6 \times 1-\mathrm{Ga}$ surface. 
which can be varied experimentally; and (2) the repulsive interaction between VLs, which is calculated from density functional theory (DFT) and verified experimentally. The lattice model connects the DFT results, calculated for perfect order, with the fluctuating disorder seen in experiment.

Experiments were carried out in ultrahigh vacuum, employing STM and low energy electron diffraction (LEED). Detailed experimental procedures for obtaining the $\mathrm{Ga} / \mathrm{Si}(112)(n \times 1)$ surface reconstruction have been reported elsewhere [6]. The $n \times 1$ periodicity was controlled by adjusting the Ga flux. All samples were equilibrated at $810 \mathrm{~K}$ for about 15 minutes and subsequently cooled to room temperature at $\sim 2 \mathrm{~K} / \mathrm{sec}$. Theoretically, within our local orbital DFT calculation (see below), the $6 \times 1$ structure is stable for $-0.6<\mu<-0.2 \mathrm{eV}$ while the $5 \times 1$ structure is stable below $-0.6 \mathrm{eV}\left(\mu_{\text {bulk }} \equiv 0\right)$. $\mu$ can be estimated from $\mu=\mu_{\text {bulk }}-k T \ln \left(p_{c} / p_{s}\right)$, where $p_{c}$ and $p_{s}$ are the Ga vapor pressures inside the effusion cell and at the sample, respectively [6].

Figures 1(a)-1(c) show three large-scale empty state STM images of the $\mathrm{Si}(112) n \times 1-\mathrm{Ga}$ surface, obtained at different $\mu$. Dark VLs meander perpendicular to the $\mathrm{Ga}$ adatom rows [6]. From these images, we identified several $m \times 1$ unit cells where $m a$ is the spacing between neighboring $\mathrm{Ga}$ vacancies along the [110] direction $(a=$ $0.384 \mathrm{~nm}$ is the lattice constant). A row of Ga atoms is adsorbed at the (111)-like step-edge sites of the $\mathrm{Si}(112)$ surface, while a second row of Ga atoms is adsorbed on the terrace sites [Fig. 1(d) and 1(e)] [6]. The resulting zigzag chains are interrupted by the meandering VLs. For each $m \times 1$ unit cell, there are $m-1 \mathrm{Ga}$ atoms on the terrace sites whereas the number of step-edge Ga atoms fluctuates between $m-2$ and $m$ [6]. Therefore, in order to acquire statistical information on the distribution of unit cell sizes, one needs to collect large-scale images and count the number of terrace atoms within each unit cell. This was most easily accomplished using empty state images (Fig. 1).

The probability distributions of the $m \times 1$ unit cells from these images are shown in Fig. 2 for different average periodicities with $5<n<6$ (i.e., different $\mu$ ). A typical sample image contained about 2000 unit cells. The vast majority of unit cells are either $5 \times 1$ or $6 \times 1$ with a small percentage of $4 \times 1$ and $7 \times 1$ unit cells. As shown, the experimental distributions can be fitted to Gaussian distribution functions with great accuracy. The Gaussian widths are practically constant $(\sigma=0.49 \pm 0.02)$ for the indicated values of $n$, conflicting with the $13 \%$ change predicted by the continuum model $[4,5]$.

Figure 3 shows the LEED line profiles along the [110] direction for various $\mu$ (and thus various $n$ ). The fractional order spots are incommensurate with the integer order reflections and shift continuously with $\mu$. They can only be indexed with three indices, instead of the two usual $(h$, $k$ ) Miller indices, $\vec{q}=(h \pm p / n) \vec{a}+k \vec{b}^{*}$, where $\bar{a}^{*}$ and $\bar{b}^{*}$ are the reciprocal lattice vectors parallel and perpendicular

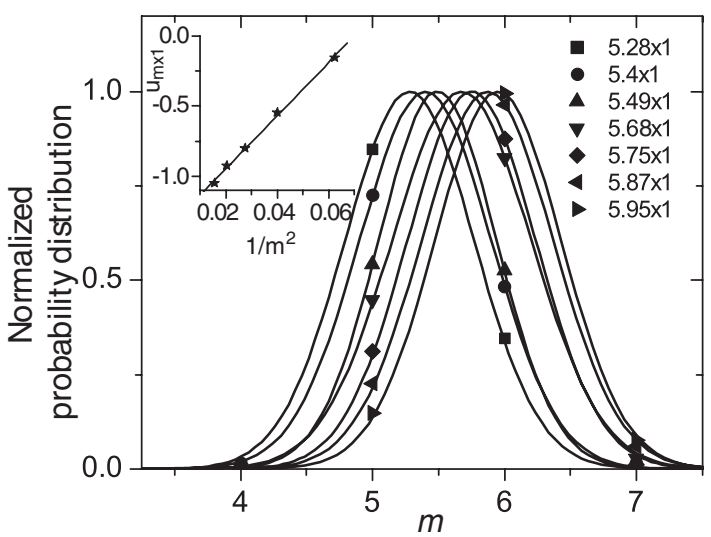

FIG. 2. Normalized distributions of $m \times 1$ unit cells on the surface, fitted with Gaussians. The centroid of Gaussian distribution shifts from $n=5.28$ to $n=5.95$ with increasing $\mu$. Their standard deviations are $\sigma=0.49 \pm 0.02$. Inset: $u_{m \times 1}$ [in eV; see Eq. (1)] versus $1 / m^{2}$ for $m=4,5,6,7$, and 8 from DFT calculations for $\mu=-0.55 \mathrm{eV}$.

to the Ga chains, respectively, and $\vec{q}$ is the momentum transfer. The fractional number $n$ represents the average VL spacing (see below) and $p$ is an integer. Evidently, varying amounts of $\mathrm{Ga}$ on the surface result in an entropic mixture of $5 \times 1$ and $6 \times 1$ units. This is consistent with an autocorrelation analysis of the VL pattern in the STM image (not shown) that shows that the sequence of $5 \times 1$ and $6 \times 1$ units along the [1 $1 \overline{0}$ ] direction is random. Indeed, the periodicity from LEED coincides with the centroids of the Gaussian fits in Fig. 2 to within $1 \%$.

Next, we analyze the distribution of unit cells in the surface by combining DFT calculations with a 1D lattice model. First, we analyze the interaction between VLs via DFT-local-density approximation (DFT-LDA) totalenergy calculations, for different periodic $m \times 1$ reconstructions ( $m=4,5,6,7$ or 8 ). From these calculations we obtain $u_{m \times 1} \equiv \Delta\left(e_{m \times 1}-n_{\mathrm{Ga}} \mu\right)$, where $e_{m \times 1}$ is total en-

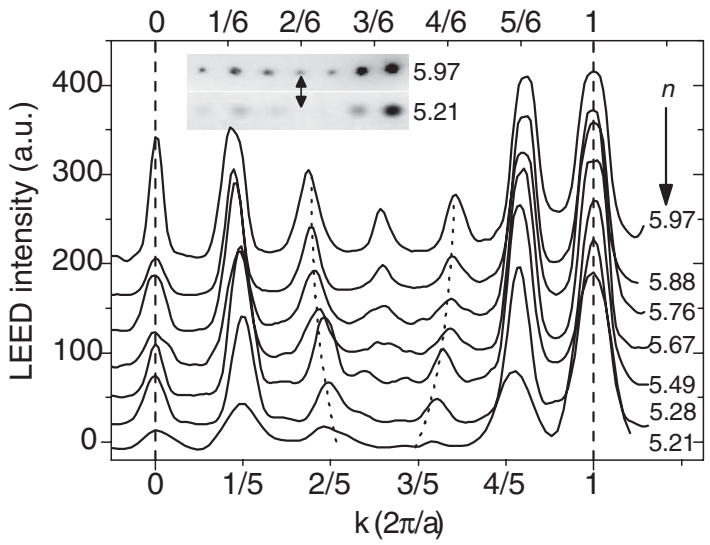

FIG. 3. LEED intensity profiles of the Ga-covered $\operatorname{Si}(112) n \times$ 1 surface $(70 \mathrm{eV})$. The average $n \times 1$ periodicities determined from the intensity profiles are indicated on the right. Dotted lines follow the continuous shifts of the fractional order spots. Inset: sections of the LEED image for $n=5.97$ and $n=5.21$. 
ergy per $m \times 1$ unit cell, and $n_{\mathrm{Ga}}=2(m-1)$ the number of Ga atoms per $m \times 1$ unit cell. The variation $\Delta$ is taken with respect to a hypothetical surface without VLs [7]. We used an efficient local orbital DFT-LDA code [8]. The use of a local orbital approach guarantees that the basis set is strictly the same in the different calculations [9]. The inset of Fig. 2 shows $u_{m \times 1}$ plotted as a function of $1 / \mathrm{m}^{2}$. The straight line indicates that the repulsive interaction between VLs is elastic in nature [10]. Accordingly [11]:

$$
u_{m \times 1}=\Lambda+G / m^{2}
$$

where $G$ defines the repulsive interaction between VLs and $\Lambda$ is the formation energy per unit cell of a VL that implicitly depends on $\mu$ [12]. From the slope of $u_{m \times 1}$ versus $1 / \mathrm{m}^{2}$, we obtain $G=19 \pm 1 \mathrm{eV}$.

In a second step we introduce a 1D lattice model in which the surface is considered as an entropic mixture of different $m \times 1$ unit cells, thus neglecting the 2D meandering along the VLs. The following analysis of the VL interactions and kink energy is conceptually different from the mean field analysis in, e.g., Ref. [4], which described the meandering of an individual VL in a mean field potential as a function of the kink energy. Here, we derive a unit cell distribution by considering an ensemble of straight lines with no kinks. Kinks are analyzed separately without reliance on a mean field model.

In our lattice model, the VLs are straight lines and the system is characterized by the numbers $N_{m}(m=4,5,6,7)$ representing the number of $4 \times 1,5 \times 1,6 \times 1$, and $7 \times 1$ unit cells of the system, and the number of VLs is $N=$ $N_{4}+N_{5}+N_{6}+N_{7}$. According to our previous discussion, we write for this 1D model:

$$
U \equiv \Delta\left(E-\mu N_{\mathrm{Ga}}\right)=N \Lambda+\sum_{m} N_{m} G / m^{2},
$$

where $E$ is the total energy of the surface and $N_{\mathrm{Ga}}=$ $6 N_{4}+8 N_{5}+10 N_{6}+12 N_{7}$ the number of $\mathrm{Ga}$ atoms on the surface. We can define the following partition function $Z$ :

$$
Z=\left(\frac{N !}{N_{4} ! N_{5} ! N_{6} ! N_{7} !}\right) \exp \left(-\frac{U}{k T}\right)
$$

and maximize $Z$ with the condition that $M=4 N_{4}+$ $5 N_{5}+6 N_{6}+7 N_{7}$ is constant (i.e., the size of the system is fixed). This yields a set of equations that give $n_{m}=$ $N_{m} / N$ as a function of $\mu$ and $T$ :

$$
\ln \left[n_{5}^{4} / n_{4}^{5}\right]=\left[\Lambda+\left(1 / 4^{2}+1 /(4 \times 5)+1 / 5^{2}\right) G\right] / k T,
$$

$$
\ln \left[n_{6}^{5} / n_{5}^{6}\right]=\left[\Lambda+\left(1 / 5^{2}+1 /(5 \times 6)+1 / 6^{2}\right) G\right] / k T,
$$

$$
\ln \left[n_{7}^{6} / n_{6}^{7}\right]=\left[\Lambda+\left(1 / 6^{2}+1 /(6 \times 7)+1 / 7^{2}\right) G\right] / k T .
$$

The numerator on the right hand side of these equations is related to $u_{m \times 1}$ for the different $m \times 1$ periodic reconstructions. For example, $6 u_{5 \times 1}-5 u_{6 \times 1}$ obtained via Eq. (1) equals the numerator on the right in Eq. (5). This result allows us to make a direct connection between the lattice model, Eqs. (4)-(6), and the DFT-LDA calculations performed for $u_{m \times 1}$.

By fitting Eqs. (4)-(6) to the distributions of $m \times 1$ unit cells observed experimentally (see Fig. 2), we determine $G / k T=570 \pm 100$ [13]. Here, the relevant temperature is the temperature $T_{F}$ below which the distribution is frozen. $T_{F}$ can be estimated from the LEED line profiles. We find that $T_{F} \approx 400 \pm 100 \mathrm{~K}$ [14]. Using this value for $T_{F}$ together with our LDA result for $G(G=19 \pm 1 \mathrm{eV})$, we obtain $G / k T=550 \pm 170$, in good agreement with the value obtained above from the fitting to Eqs. (4)-(6). From the fitting, we find that the width of the distribution depends only on the ratio $G / k T$ whereas the average periodicity depends on both $G / k T$ and $\mu$ [13]. Specifically, $\mu$ varies by $0.32 \pm 0.08 \mathrm{eV}$ for $5.21 \leq n \leq$ 5.95 , in good agreement with our local orbital calculations showing a variation in $\mu$ of $0.26 \mathrm{eV}$.

We finally turn our attention to the 2D meandering behavior and determination of the kink energy. We restrict our analysis to the case $n=5.49$ : in this case the surface contains almost equal amounts of $5 \times 1$ or $6 \times 1$ unit cells, with a negligible number of $4 \times 1$ and $7 \times 1$ unit cells (out of $N=3461$ unit cells analyzed we found only six $4 \times 1$ and five $7 \times 1$ unit cells), and will be neglected in the following analysis.

A vacancy can be located in between a $5 \times 1$ and $6 \times 1$ unit cells, a configuration which we denote as $\frac{5}{6}$; other configurations are $\frac{6}{5}, \frac{6}{6}$ and $\frac{5}{5}$. Similarly, the kinks in the VLs can be classified according to the configuration of the four surrounding unit cells, as, e.g., $\frac{6}{5} \frac{5}{6}$, Fig. 4(a), or $\frac{6}{6} \frac{6}{5}$, Fig. 4(b), etc. Only kinks of the type $\frac{6}{5} \frac{5}{6}$ or $\frac{5}{6} \frac{6}{5}$ appear as regular kinks: all other kink configurations imply the presence of a correlated kink in a neighboring VL, as indicated by the circles in Fig. 4(b). Thus, we use the terms regular
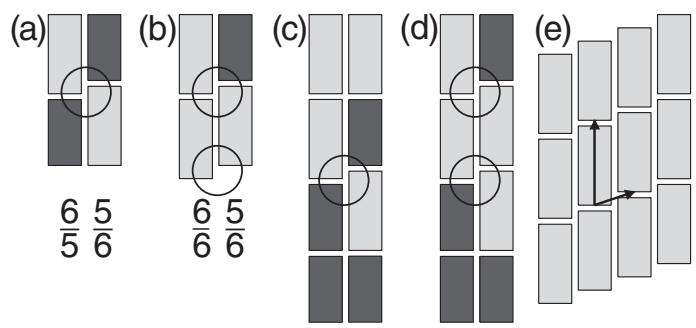

FIG. 4. Schematic drawing of different kink configurations. Light/dark rectangles represent $6 \times 1 / 5 \times 1$ unit cells. (a) Regular kink, (b) nonregular kinks (see text). (c) and (d) Two possible configurations for the model system used in our analysis. Circles in (a)-(d) highlight kink positions. (e) Shows a portion of the oblique lattice used in the DFT calculations of the kink energy (oblique lattice vectors indicated by arrows). 
kink, to refer to $\frac{6}{5} \frac{5}{6}$ or $\frac{5}{6} \frac{6}{5}$ kinks, and nonregular kink for the others. Experimentally, we counted the total number of kinks, $N_{K}$, as well as the number of regular $N_{R}$ and nonregular $N_{\mathrm{NR}}$ kinks, obtaining $N_{K} / N=0.060$ and $N_{\mathrm{NR}} / N_{R}=0.20$. If all kinks were created independently, as implicitly assumed in mean field models of VLs [4], then the ratio $N_{\mathrm{NR}} / N_{R}$ should have been 0.13 for the present case with $N_{K} / N=0.06$. The $\sim 50 \%$ higher fraction of nonregular kinks observed here implies correlated meandering, meaning that kink energies cannot be calculated reliably using the mean field model [4].

Based on the experimentally observed absence of ordering of the different $m \times 1$ unit cells in the [110] direction, we analyzed the number of one-step kinks, $N_{K}$, along a VL using the model system depicted in Figs. 4(c) and 4(d). In this model system of $4 \times 2=8$ unit-cells, we consider all $2^{8}=256$ possible configurations of $5 \times 1$ and $6 \times 1$ unit cells; the probability of each is proportional to $\exp \left(-n_{k} e_{k} / k T_{F}\right)$ where $n_{k}$ is the number of kinks it contains and $e_{k}$ the kink energy. We find that the ratios $N_{K} / N$ and $N_{\mathrm{NR}} / N_{R}$ are already converged for this small-size model system. From this model we obtain $N_{K} / N$ as a function of $\exp \left(-e_{k} / k T_{F}\right)$, and deduce the kink energy $e_{k}$ using the experimental ratio $N_{K} / N=$ 0.06 and $T_{F}=400 \mathrm{~K}$. This yields $e_{k}=73 \pm 20 \mathrm{meV}$. From this model we can also obtain $N_{\mathrm{NR}} / N_{R}$ as a function of $\exp \left(-e_{k} / k T_{F}\right)$, and obtain an independent estimate of the kink energy. This procedure yields $e_{k}=68 \pm 20 \mathrm{meV}$, showing the consistency of this analysis (the errors mainly arise from the large uncertainty in $T_{F}$ ). We also estimated the kink energy $e_{k}$ by means of DFT calculations for a periodic $6 \times 1$ and $5 \times 1$ arrangement of kinks using oblique lattice vectors; see Fig. 4(e). These calculations yield $e_{k}=80 \pm 10 \mathrm{meV}$ and $e_{k}=88 \pm 10 \mathrm{meV}$, respectively, in excellent agreement with the experimental values above. Note that a mean field analysis using $N_{K} / N=0.06$ would have produced a higher kink energy of $119 \pm$ $30 \mathrm{meV}$. This overestimation is consistent with the fact that vacancy correlations are not included in the 1D mean field model, although they effectively suppress thermal meandering.

In summary, the self-organization process of atom vacancies in the $\mathrm{Ga} / \mathrm{Si}(112)$ interface can be experimentally controlled via the chemical potential of the adsorbate. A new lattice model accurately connects first principles DFT calculations for ordered structures with experimental data from disordered structures, and naturally incorporates the chemical potential. This hybrid approach of lattice modeling and DFT may be useful to other self-organization processes where fluctuations are bound by discrete length scales in the system.

This work is funded in part by the Stichting voor Fundamenteel Onderzoek der Materie (FOM), which is financially supported by the Nederlandse Organisatie voor Wetenschappelijk Onderzoek (NWO), the National Science Foundation under Contract No. DMR 0244570, and by the Ministerio de Educación y Ciencia (Spain) under project No. MAT2004-01271. H. H.W. also acknowledges support from the U.S. Department of Energy, Office of Basic Energy Sciences, Division of Materials Sciences and Engineering. Oak Ridge National Laboratory is managed by UT-Battelle, LLC.

*Electronic address: snijderspc@ ornl.gov

Present address: Materials Science and Technology Division, Oak Ridge National Laboratory, Oak Ridge, TN 37831, USA

[1] W. D. Knight et al., Phys. Rev. Lett. 52, 2141 (1984); G. Springholz et al., Science 282, 734 (1998); H. Brune et al., Nature (London) 394, 451 (1998).

[2] S. C. Erwin et al., Phys. Rev. Lett. 83, 1818 (1999).

[3] F. Liu, F. Wu, and M. G. Lagally, Chem. Rev. 97, 1045 (1997); B. Voigtländer, Surf. Sci. Rep. 43, 127 (2001).

[4] X. Chen et al., Phys. Rev. Lett. 73, 850 (1994).

[5] N. C. Bartelt, T. L. Einstein, and E. D. Williams, Surf. Sci. 276, 308 (1992).

[6] C. González et al., Phys. Rev. Lett. 93, 126106 (2004); P. C. Snijders et al., Phys. Rev. B 72, 125343 (2005).

[7] i.e., $\quad u_{m \times 1}=\left(e_{m \times 1}-2(m-1) \mu\right)-\left(m e_{1 \times 1}-2 m \mu\right)=$ $e_{m \times 1}-m e_{1 \times 1}+2 \mu$, where $e_{1 \times 1}$ is the total energy for a $1 \times 1$ unit cell (surface without VLs).

[8] A. A. Demkov et al., Phys. Rev. B 52, 1618 (1995). Details for the present calculations can be found in [6].

[9] The DFT analyses are based on total-energy comparisons for different periodicities. Similar calculations using a plane-wave code would require very large basis sets because the cancellation of errors implicit in the calculation of total-energy differences is only effective when comparing calculations with identical basis sets.

[10] V. I. Marchenko and A. Ya. Parshin, Sov. Phys. JETP 52, 129 (1980); entropic repulsions can be ignored; see, e.g., H. J. W. Zandvliet, Rev. Mod. Phys. 72, 593 (2000).

[11] C. V. Ciobanu, D. T. Tambe, and V. B. Shenoy, Surf. Sci. 556, 171 (2004).

[12] $\Lambda$ depends on $\mu$ as $\Lambda=\Omega+2 \mu$ (there are two Ga vacancies per unit cell), where $\Omega$ is a constant.

[13] Equations (4)-(6) represent near-perfect Gaussians, showing only a marginal skewness to high $n$; this is most clearly found near $n=5.5$. Direct data fitting to Eqs. (4)-(6) is unreliable because the tail of the distribution only consists of one data point, $n_{4}$ or $n_{7}$; both are subject to large statistical fluctuations. Therefore, motivated by the experimental observations, we first fitted Eqs. (4)-(6) to Gaussian distributions and then compared the widths of the theoretical Gaussians to the experimental ones. This produces an analytical expression that relates $\sigma$ to $G / k T ; \mu$ drops out.

[14] We observe significant narrowing of the LEED line profile above $500 \mathrm{~K}$, indicating that spatial fluctuations start to randomize within the transfer width of the LEED system. This sets the upper limit for the freezing temperature. The lower limit is the temperature at which the STM data were recorded, i.e., room temperature. 